University of Nebraska - Lincoln

DigitalCommons@University of Nebraska - Lincoln

Ralph Skomski Publications

Research Papers in Physics and Astronomy

2009

Graded Permanent Magnets

Ralph Skomski

University of Nebraska-Lincoln, rskomski2@unl.edu

J. Hadjipanayis

University of Delaware, Newark, DE

David J. Sellmyer

University of Nebraska-Lincoln, dsellmyer@unl.edu

Follow this and additional works at: https://digitalcommons.unl.edu/physicsskomski

Part of the Physics Commons

Skomski, Ralph; Hadjipanayis, J.; and Sellmyer, David J., "Graded Permanent Magnets" (2009). Ralph Skomski Publications. 59.

https://digitalcommons.unl.edu/physicsskomski/59

This Article is brought to you for free and open access by the Research Papers in Physics and Astronomy at DigitalCommons@University of Nebraska - Lincoln. It has been accepted for inclusion in Ralph Skomski Publications by an authorized administrator of DigitalCommons@University of Nebraska - Lincoln. 


\title{
Graded permanent magnets
}

\author{
R. Skomski, ${ }^{1, a)}$ G. C. Hadjipanayis, ${ }^{2}$ and D. J. Sellmyer ${ }^{1}$ \\ ${ }^{1}$ Nebraska Center for Materials and Nanoscience and Department of Physics and Astronomy, \\ University of Nebraska, Lincoln, Nebraska 68588, USA \\ ${ }^{2}$ Department of Physics and Astronomy, University of Delaware, Newark, DE 19716
}

(Presented 13 November 2008; received 30 August 2008; accepted 7 November 2008; published online 16 March 2009)

The effect of semihard magnetic phases and interfaces on the performance of nanostructured two-phase permanent magnets is investigated by model calculations. In addition to the trivial coercivity increase due to the replacement of soft regions by semihard regions, there is a coercivity enhancement even if the volume-averaged anisotropy is kept constant during the introduction of the semihard phase. A variational approach is used to derive analytical results for representative anisotropy profiles. The improvement is operative on length scales slightly larger than that of the soft phase in hard-soft composites, but the main challenge is to find semihard light or heavy transition metal phases with a high magnetization. There are several Fe- and Co-based phases, but most are thin-film systems and difficult to use in bulk magnets. Very hard nanostructured magnets may also be created from soft phases with negative but large anisotropy constants (hard-magnetic soft-soft magnets). (C) 2009 American Institute of Physics. [DOI: 10.1063/1.3068622]

\section{INTRODUCTION}

The energy product of aligned two-phase magnets ${ }^{1-3}$ exhibits a practical limitation due to the dependence of the coercivity on the local anisotropy $K_{1}(\mathbf{r})$, so that theoretical energy products are difficult to achieve. In two-phase permanent magnets, which are usually composed of hard and soft phases, the main role of the soft phase is to enhance the magnetization. However, the soft phase negatively affects coercivity and energy product, and the use of semihard phases is a potential approach to stabilize the coercivity. Arbitrary anisotropy profiles were first discussed in the mid1990s (Ref. 4) but have recently attracted renewed attention from an experimental point of view ${ }^{5,6}$ as a tool to improve energy product and coercivity. Arbitrary concentration profiles are also of interest in magnetic recording, where graded media may be used to control coercivity and to facilitate the writing process. ${ }^{7,8}$ Last but not least, semihard phases may be useful to reduce the rare-earth content of permanent magnets, which is a major challenge in present-day permanentmagnet research.

Exchange coupling in nanostructures of arbitrary anisotropy profiles $K_{1}(\mathbf{r})$, including semihard phases, involves the correlation function $\left\langle K_{1}(\mathbf{r}) K_{1}\left(\mathbf{r}^{\prime}\right)\right\rangle$ (Ref. 4) and is therefore strongly real-structure dependent. The replacement of a soft phase by a semihard phase yields a trivial improvement of the coercivity because the average anisotropy $\left\langle K_{1}\right\rangle$ increases. However, the coercivity also improves if the volumeaveraged anisotropy is kept constant, that is, by using the semihard material to simultaneously replace hard and soft regions or by creating smooth interfaces. In lowest order, this enhancement is described by $\left\langle K_{1}(\mathbf{r}) K_{1}\left(\mathbf{r}^{\prime}\right)\right\rangle$, which enters the equation for the nucleation field (coercivity) and yields correction to the Stoner-Wohlfarth prediction $H_{c}$

${ }^{a)}$ Electronic mail: rskomski@neb.rr.com. $=2\left\langle K_{1}\right\rangle / \mu_{o} M_{s}{ }^{4}{ }^{4}$ This paper uses a simple nanoparticle model to analyze the hardening effect due to a semihard phase and discusses some material requirements for the semihard phase.

\section{CALCULATION AND RESULTS}

While the findings of the paper are very general, it is useful to consider a well-defined and physically transparent system. Let us consider cylindrical nanoparticles of length $L$, Fig. 1, with arbitrary but monotonic anisotropy profiles $K_{1}(z)$. The average anisotropy is kept constant during structural variation, $\left\langle K_{1}(z)\right\rangle=K_{h} / 2$, so that all structures yield the same Stoner-Wohlfarth or coherent-rotation limit $H_{c}$ $=K_{h} / \mu_{o} M_{s}$, but the profiles near the interface are very different. As elaborated elsewhere, ${ }^{2,9}$ the nucleation modes of nanoparticles such as those shown in Fig. 1 obey the partial differential equation

$$
-A \nabla^{2} m+\left[K(\mathbf{r})-\mu_{o} M_{s} H / 2\right] m=0,
$$

where the effective lowest-order uniaxial anisotropy constant $K(\mathbf{r})$ describes the real or defect structure of the magnet. $K$ also contains the magnetostatic self-interaction, which is a good approximation unless flux closure (curling) is

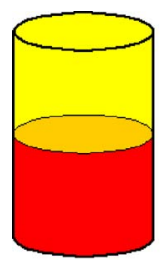

(a)

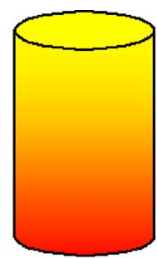

(b)

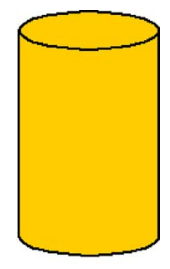

(c)
FIG. 1. (Color online) Cylindrical particles with inhomogeneous magnetization profiles: (a) hard-soft composite, (b) graded interface, and (c) semihard magnet. Dark and bright regions correspond to high and low anisotropies, respectively. 


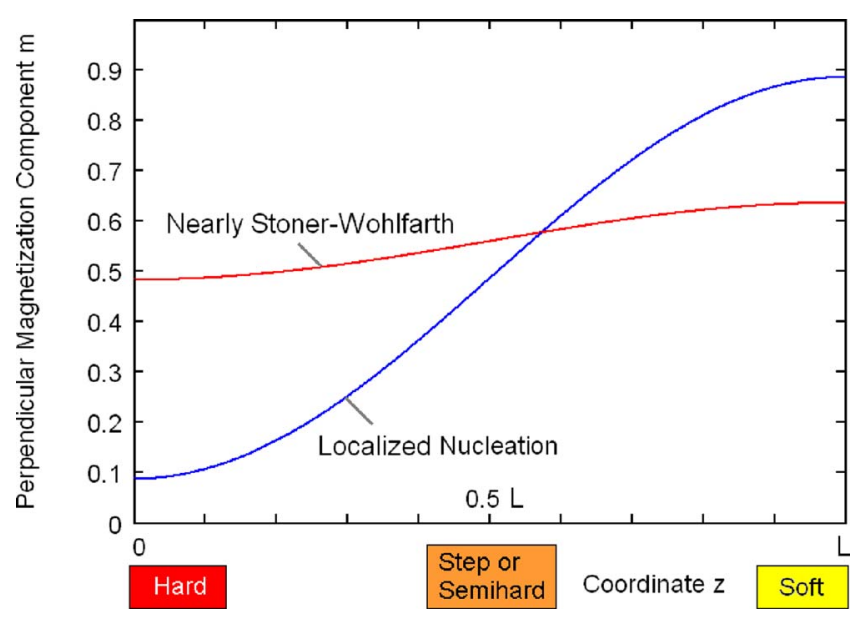

FIG. 2. (Color online) Spatial extension of nucleation mode, illustrating the transition from coherent rotation to localized nucleation. The limit of Stoner-Wohlfarth reversal (coherent rotation) corresponds to Fig. 1(c) but is also realized in very small particles irrespective of anisotropy profile.

important. ${ }^{9}$ For homogeneous ellipsoids of revolution, $K(\mathbf{r})$ $=\langle K\rangle$, and Eq. (1) reproduces coherent rotation irrespective of domain-wall width and particle size. Coherent rotation is also realized for very small particles because the exchangeenergy density scales as $A / L^{2}$ and magnetization inhomogeneities are very unfavorable in small magnets. We therefore expect the structures of Fig. 1 to exhibit a common coercivity $H_{c}=K_{h} / \mu_{o} M_{s}$ in the limit of small cylinder lengths $L$.

To perform the calculation, we have used a variational method very similar to that on p. 281 in Ref. 10, which amounts to the diagonalization of a $2 \times 2$ matrix derived from Eq. (1). This matrix is constructed from the uniform mode $m=$ const and forms the lowest-lying sinusoidal mode $m^{\prime}=m_{o} \cos (\pi z / L)$. For homogeneous cylinders, Fig. 1(c), the uniform mode $m$ has the lowest energy, but inhomogeneities of the symmetry of Figs. 1(a) and 1(b) yield nondiagonal matrix elements between $m$ and $m^{\prime}$. Physically, the applied field favors a magnetization reversal that starts in the soft regions, and the corresponding mode is realized by admixing some $m^{\prime}$ character to $m$. Figure 2 shows two nucleation modes and illustrates how the present model interpolates between coherent rotation and localized nucleation.

Explicit results are shown for the three anisotropies of Fig. 1, namely, two-phase particles containing 50\% hard phase $\left(K_{1}=K_{h}\right)$ and $50 \%$ soft phase $\left(K_{1}=K_{s}=0\right)$, particles with a linear end-to-end variation from to $K_{h}$ to 0 , and homogeneous particles of anisotropy $K_{1}=K_{h} / 2$. Other profiles have also been examined, but the results are essentially intermediate between the first two profiles. Figure 3 shows explicit nucleation fields for the three cylindrical model particles. As mentioned above, these choices all conserve the average anisotropy $\left\langle K_{1}(\mathbf{r})\right\rangle$ and ensure a common StonerWohlfarth coercivity maximum $H_{c}=K_{1} / \mu_{o} M_{s}$ in the limit of very small particles.

\section{SEMIHARD PHASES}

The use of semihard materials and grain boundaries helps ensure coercivity, but the magnetization of the known semihard phases is much lower than that of soft phases such

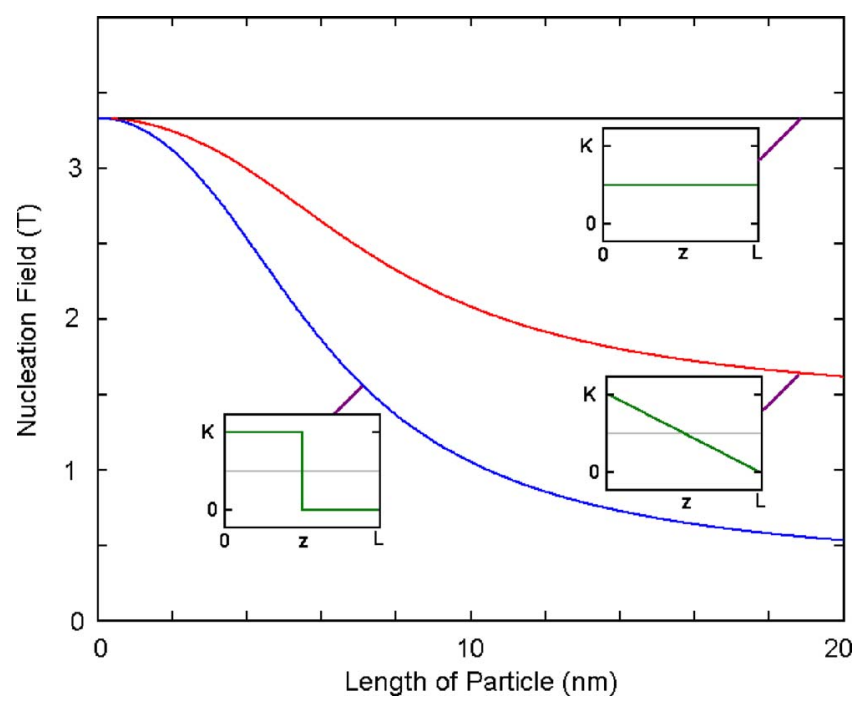

FIG. 3. (Color online) Nucleation field for several concentration profiles $K_{1}(\mathbf{r})$. The three profiles have a common average anisotropy $\left\langle K_{1}(\mathbf{r})\right\rangle$ $=K_{h} / 2$ but different nucleation fields. The cases shown in this figure correspond to Fig. 1.

as $\mathrm{Fe}$ and $\mathrm{Fe}-\mathrm{Co}$. It may also be useful to reduce material costs by reducing the rare-earth content. In fact, the original emphasis on soft phases ${ }^{2}$ had its origin in the role of the magnetization $M_{s}$ as a limiting factor in energy product development, $(B H)_{\max } \leq \mu_{o} M_{s}^{2} / 4$. Semihard phases were not considered at that time because their magnetizations are roughly comparable to that of $\mathrm{Nd}_{2} \mathrm{Fe}_{14} \mathrm{~B}\left(\mu_{o} M_{s}=1.61 \mathrm{~T}\right)$. In fact, there is no point in replacing a hard region by a semihard region just to avoid the use of soft-magnetic material for coercivity reasons.

Aside from important practical considerations such as (non)equilibrium phase formation and suitable interface and nanostructure, the challenge is therefore to find semihard materials that combine moderate anisotropy with a high magnetization. $\mathrm{Fe}_{1-x} \mathrm{Co}_{x}$ alloys are ideal candidates for hard-soft nanostructuring because they have a very high magnetization in a wide range of Fe-rich compositions, $2.43 \mathrm{~T}$ in $\mathrm{Fe}_{65} \mathrm{Co}_{35} \cdot{ }^{11,12}$ However, it is difficult to turn these rather soft materials into semihard phases. Burkert et al. ${ }^{13}$ predicted substantial $K_{1}=9.5 \mathrm{MJ} / \mathrm{m}^{3}$ and $\mu_{o} M_{s}=1.9 \mathrm{~T}$ for tetragonally distorted Fe-Co with $c / a=1.23$. This is better than hcp Co, where $K_{1}=0.5 \mathrm{MJ} / \mathrm{m}^{3}$ and $\mu_{o} M_{s}=1.76 \mathrm{~T}$, but such large strains are hardly practical. Experimental roomtemperature anisotropies per $\mathrm{Fe}$ or $\mathrm{Co}$ atom reach about $2.1 \mathrm{MJ} / \mathrm{m}^{3}$, but this value does not account for the large amount of Pt (about $75 \mathrm{vol} . \%$ ) in the layer necessary to stabilize these structures. ${ }^{14}$

A potentially very useful semihard material is $\mathrm{Co}_{3} \mathrm{Pt}$ with experimental anisotropies of the order of $2 \mathrm{MJ} / \mathrm{m}^{3}$ $\left(20 \mathrm{ergs} / \mathrm{cm}^{3}\right)$. The orthorhombic Pmm 2 phase of $\mathrm{Co}_{3} \mathrm{Pt}$ has an anisotropy of about $2.8 \mathrm{MJ} / \mathrm{m}^{3}\left(28 \mathrm{ergs} / \mathrm{cm}^{3}\right)$, but its equilibrium formation requires a strain of more than $1 \% .^{15} \mathrm{~A}$ system with very high interface anisotropies is $\mathrm{Fe} / \mathrm{W}(110)$. For some structures, the predicted anisotropy reaches $6 \mathrm{meV}$ per Fe atom, ${ }^{16}$ and large $\mathrm{W}$ orbital moments indicate that the spin-orbit coupling of the $\mathrm{W}$ is important to understand the magnetization and anisotropy of the system. As in other sys- 

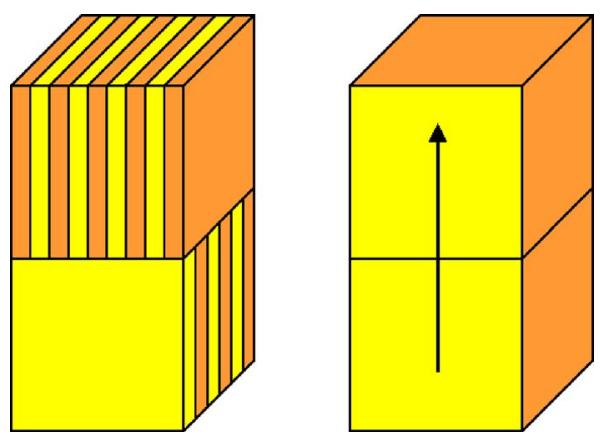

FIG. 4. (Color online) Easy-axis magnetism in exchange-coupled easyplane magnets (HMSS magnets). Two nanoscale easy-plane magnets with $K_{1}=-K_{o}$ are exchange coupled at an angle of $90^{\circ}$ (left), which yields an easy axis magnet with $K_{1}=K_{o} / 2$.

tems, it is challenging to exploit this high anisotropy in bulk magnets. Usable hard-magnetic properties may also be created by doping or coatings using heavy transition metals, such as Pt and W.

It is known that the size of the soft phases should not be larger than about twice the domain-wall width $\delta_{B}=\pi \delta_{o}$ of the hard phase, where $\delta_{o}=\left(A / K_{h}\right)^{1 / 2}$. For semihard phases, $\delta_{o}$ must be replaced by $\left[A /\left(K_{h}-K_{s}\right)\right]^{1 / 2}$, where $K_{s}$ is the anisotropy of the hard or soft phase. This means that the semihard phases are slightly more forgiving as soft phases as far a length-scale requirements are concerned.

An example of rather unconventional nanostructuring is hard-magnetic soft-soft (HMSS) composites. The idea, shown in Fig. 4, is to use two ferromagnetic easy-plane materials with negative but large anisotropy $K_{1}=-K_{o}$, which are exchange coupled to each other at an angle of $90^{\circ}$ between the $c$ axes. On a nanoscale, this yields an effective anisotropy constant $K_{o} / 2$, that is, a material that is very hard though not as hard as a single-phase material with positive $K_{1}$. For example, in the bottom-left part or 'grain' in Fig. 4, the easy magnetization plane (visualized by schematic atomic layers) is in the paper plane, but the corresponding free magnetization rotation is inhibited by the top-left grain whose easy plane is perpendicular to the paper plane. There exist materials with very large negative $K_{1}$, but some of these are antiferromagnets for which the simple picture of Fig. 4 does not work. An example of an antiferromagnet with very strong easy-plane anisotropy is $\operatorname{MnIr}\left(K_{1}=-21 \mathrm{MJ} / \mathrm{m}^{3}\right){ }^{17}$ An experimental approach would be to start from an easyplane magnet and to use mechanical alloying (ball milling) to create oblique nanoscale interfaces. For example, $\mathrm{Sm}_{2} \mathrm{Co}_{14} \mathrm{~B}$, which has a room-temperature magnetization of $1.51 \mathrm{~T}$ and an anisotropy of $-12.0 \mathrm{MJ} / \mathrm{m}^{3},{ }^{18}$ compared to $+4.9 \mathrm{~T}$ for $\mathrm{Nd}_{2} \mathrm{Fe}_{14} \mathrm{~B}$, would then yield a fairly hard material. Finally, there is renewed interest in $\mathrm{Fe}_{16} \mathrm{~N}_{2}$, which has a very high magnetization and some anisotropy but is very difficult to produce in the bulk form. ${ }^{19}$

\section{CONCLUSIONS}

In summary, we have investigated how semihard phases and interfaces affect coercivity and energy product of aligned two-phase magnets. If structured on an appropriate length scale, the coercivity improvement goes beyond the trivial improvement due to the replacement of soft-magnetic phase by a semihard phase. However, the main challenge is to find a suitable semihard phase with a magnetization significantly larger than about 1.6 T. Another approach to create hardmagnetic materials is to exchange couple soft materials that have a strongly negative anisotropy constant.

\section{ACKNOWLEDGMENTS}

This research is supported by DOE (DE-FG0204ER46152) and NCMN.

${ }^{1}$ E. F. Kneller and R. Hawig, IEEE Trans. Magn. 27, 3588 (1991). ${ }^{2}$ R. Skomski and J. M. D. Coey, Phys. Rev. B 48, 15812 (1993).

${ }^{3}$ P. Liu, C. P. Luo, Y. Liu, and D. J. Sellmyer, Appl. Phys. Lett. 72, 483 (1998).

${ }^{4}$ R. Skomski and J. M. D. Coey, IEEE Trans. Magn. 30, 607 (1994).

${ }^{5}$ J. S. Jiang, J. E. Pearson, Z. Y. Liu, B. Kabius, S. Trasobares, D. J. Miller, S. D. Bader, D. R. Lee, D. Haskel, G. Srajer, and J. P. Liu, Appl. Phys. Lett. 85, 5293 (2004).

${ }^{6}$ G. Asti, M. Ghidini, M. Solzi, and F. M. Neri, Proceedings of the HPMA, 2004, edited by N. M. Dempsey and P. de Rango (unpublished), p. 442. ${ }^{7}$ D. Suess, Appl. Phys. Lett. 89, 113105 (2006).

${ }^{8}$ R. Skomski, T. A. George, and D. J. Sellmyer, J. Appl. Phys. 103, 07F531 (2008).

${ }^{9}$ R. Skomski, J. Phys.: Condens. Matter 15, R841 (2003).

${ }^{10}$ R. Skomski, Simple Models of Magnetism (University Press, Oxford, 2008).

${ }^{11}$ R. M. Bozorth, Ferromagnetism (van Nostrand, Princeton, NJ, 1951).

${ }^{12}$ R. Skomski and J. M. D. Coey, Permanent Magnetism (Institute of Physics, Bristol, 1999).

${ }^{13}$ T. Burkert, L. Nordström, O. Eriksson, and O. Heinonen, Phys. Rev. Lett. 93, 027203 (2004).

${ }^{14}$ G. Andersson, T. Burkert, P. Warnicke, M. Björck, B. Sanyal, C. Chacon, C. Zlotea, L. Nordström, P. Nordblad, and O. Eriksson, Phys. Rev. Lett. 96, 037205 (2006).

${ }^{15}$ S. D. Willoughby, R. A. Stern, R. Duplessis, J. M. MacLaren, M. E. McHenry, and D. E. Laughlin, J. Appl. Phys. 93, 7145 (2003).

${ }^{16}$ T. Andersen and W. Hübner, Phys. Rev. B 74, 184415 (2006).

${ }^{17}$ R. Y. Umetsu, A. Sakuma, and K. Fukamichi, Appl. Phys. Lett. 89, 052504 (2006).

${ }^{18}$ Rare-Earth Iron Permanent Magnets, edited by J. M. D. Coey (University Press, Oxford, 1996).

${ }^{19}$ S. G. Sankar, private communication. 